\title{
Multiple-vacancy production in the independent-Fermi-particle model
}

\author{
R. L. Becker \\ Physics Division, Oak Ridge National Laboratory, Oak Ridge, Tennessee 37831 \\ A. L. Ford and J. F. Reading \\ Physics Department, Texas A\&M University, College Station, Texas 77843
}

(Received 12 January 1984)

\begin{abstract}
Measurements of $K \mathrm{x}$-ray (or Auger) satellite intensities produced by ion impact have yielded $K^{u} L^{v}$ multiple-vacancy distributions which in most cases are nearly binomial. This implies that, statistically, the holes are nearly independent, which is surprising in view of the known influence of correlations on other collision processes. We give a detailed derivation of general expressions for ion-induced multiple-vacancy distributions in the independent-Fermi-particle collision model, based on the Hartree-Fock description of the target, which contains Pauli correlations. Our coupledchannels calculations employing these expressions have shown that the electron exchange terms tend to mutually cancel because of "random" phases, but need not cancel when a single channel, such as a resonant electron capture, is dominant.
\end{abstract}

\section{INTRODUCTION}

Most atomic collision theory involves an independentparticle model (IPM) for the electrons. The IPM for electrons involved in collisions should employ an antisymmetric many-electron wave function, just as it does for bound states in the Hartree-Fock (HF) approximation. However, all the theory ${ }^{1}$ of $K$-shell-vacancy plus multiple- $L$-shell-vacancy production in ion-atom collisions, ${ }^{2}$ previous to our work beginning with Ref. 3 , has been done with a model in which one forms transition probabilities for a many-electron system directly from the probabilities of a one-electron system. Because in this model quantum mechanics is not used except for a oneelectron system, we refer to it as the single-particle model (SPM). The Pauli exclusion principle (only in the original sense of excluding multiple occupancy of a spin orbital, and not in the stronger sense of Dirac's requirement of antisymmetry of the wave function) is imposed on the SPM, so that a given spin orbital $k$ is either vacant, with a probability $p_{k}$, or occupied by precisely one electron, with probability $1-p_{k}$. Then simple counting leads to a binomial distribution for the numbers of vacancies. Some currency has been obtained for an alternative designation of this model, namely, the "independent-electron approximation," which appropriately emphasizes that its multiple-vacancy formulas contain no correlation terms.

An alternative derivation ${ }^{4}$ of the binomial distribution has been given, which is based on the minimum crossentropy principle of information theory. Just as in the SPM, given a set of $N$ spin orbitals, e.g., the $L$ shell with $N=8$, one imposes the weak form of the Pauli exclusion principle, that each spin orbital may be either vacant or occupied by only one electron. There are then $2^{N}$ possible final many-electron states, $\kappa$, which can be made from this set, each of which has a certain number, $N_{\kappa}^{\prime}=0,1, \ldots, N$ of the spin orbitals occupied. Whether the states $\kappa$ containing more than one electron are antisymmetrical or not is irrelevant to the derivation. One assumes that the Bayesian prior probability distribution of the final states $\kappa$ is uniform, $p_{\kappa}^{(0)}=2^{-N}$. Then the entropy principle leads to the posterior distribution

$$
p_{\kappa}=(1-p)^{N_{\kappa}^{\prime}} p^{N-N_{\kappa}^{\prime}}, p \equiv\left\langle N-N^{\prime}\right\rangle / N
$$

where $p$ is the mean vacancy probability per spin orbital, which can be measured. Then the probability of $N^{\prime}$ occupancies and $N-N^{\prime}$ vacancies is

$$
P_{N-N^{\prime}}^{N^{\prime}}=\sum_{\substack{\kappa=1 \\
\left(N_{\kappa}^{\prime}=N^{\prime}\right)}}^{2^{N}} p_{\kappa}=\left[\begin{array}{l}
N \\
N^{\prime}
\end{array}\right](1-p)^{N^{\prime}} p^{N-N^{\prime}},
$$

the binomial distribution. This derivation indicates that even in the presence of correlations in the wave functions, the binomial distribution is the most probable, given only the known constraints. Whether the detailed dynamics (shell structure, form of the interaction, charge and speed of the projectile ion, etc.) lead effectively to additional constraints, not yet taken into account, cannot be answered by the statistical theory.

In contrast to the SPM our dynamical theory includes hole-hole correlations generated by using an antisymmetric wave function. To stress that when the IPM employs a many-electron wave function antisymmetric under exchange, i.e., when it is a Slater determinant,

$$
\begin{aligned}
& \Psi^{\mathrm{IFPM}}\left(\overrightarrow{\mathrm{r}}_{1}, \ldots, \overrightarrow{\mathrm{r}}_{F}, t\right)=(F !)^{-1 / 2} \operatorname{det}\left[\psi_{\alpha i}\right], \\
& \psi_{\alpha i}=\psi_{\alpha}\left(\overrightarrow{\mathrm{r}}_{i}, t\right), \quad \alpha, i=1,2, \ldots, F,
\end{aligned}
$$

the electrons are treated properly as indistinguishable Fermions, we refer to this formulation as the independentFermi-particle model (IFPM). In the IFPM the electrons remain dynamically independent but have Pauli correlations arising from the Fermi statistics. One of us has considered elsewhere ${ }^{5}$ the independent-distinguishableparticle model (IDPM), which employs a many-electron 
wave function taken to be a simple product of spin orbitals, without electron exchange, as in the old Hartree atomic theory. Unlike the SPM, the IDPM multiplevacancy formulas contain correlation terms, arising from multiple occupancy of final spin orbitals, but these physically spurious terms are entirely incoherent in contrast to those of the IFPM. In the IFPM such incoherent terms are canceled by similar ones arising from electron exchange.

By an exclusive reaction we shall mean one leading to a unique final state. Most experiments in atomic collision physics detect inclusive processes, i.e., a set of exclusive reactions which have something in common, such as the production of a hole in a given state or of a free electron. In the context of the IPM we shall distinguish between particular inclusive processes, for which all the final states must contain some particular spin orbitals, and hyperinclusive processes, which involve sums over particular inclusive processes such that the specified spin orbitals must belong only to some set, such as a shell. As an example, we cite inclusive $L$-shell-vacancy production, the set of reactions leading to at least one hole in the $L$ shell.

In the IPM it is easy to sum over all the reactions of an inclusive process, and the resulting cross section is usually given by a much simpler expression than that for one of its exclusive components. ${ }^{6}$ In the case of a "singles" measurement, such as electron capture from an atom to a particular state of an initially bare projectile, the IFPM gives the same inclusive cross section as does the SPM. We shall employ a classical treatment of the relative motion of the projectile and target nuclei, so that the integrated cross section for a particular inclusive process is related to a corresponding impact parameter-dependent probability, $P(b)$ or $\rho(b)$, by

$$
\sigma=2 \pi \int_{0}^{\infty} P(b) b d b .
$$

For a hyperinclusive process sometimes instead of the probability in (1.2) one has an expected value, $Q(b)$, which may be greater than one. For example, the expected value for capture into the $K$ shell of the projectile is

$$
Q^{C(K)}(b)=\rho^{C(1 s \uparrow)}(b)+\rho^{C(1 s \downarrow)}(b) \leq 2 .
$$

The IFPM inclusive probability for occupancy of a spin orbital $k$ is $^{6}$

$$
\rho^{k}(b)=\sum_{h}^{\text {occ }}\left|a_{k h}(b)\right|^{2} \equiv a_{k k}^{(2)}(b)
$$

and for a vacancy is

$$
\rho_{k}(b)=1-\rho^{k}(b)
$$

where $a_{k h}(b)$ is the amplitude of the single-electron transition from spin orbital $h$ at $t \rightarrow-\infty$ to $k$ at $t \rightarrow \infty$ and $h$ runs over all the initially occupied spin orbitals. Thus $\rho^{k}(b)$ is just the incoherent sum of single-electron probabilities for transitions from all the initially occupied spin orbitals to the final state $k$. This result is obtained by summing over all possible final states for the $F-1$ other electrons. Our notation will, in general, list occupancies as superscripts and nonoccupancies as subscripts.

For a "doubles" measurement, i.e., a twofold coin- cidence, the IFPM inclusive cross section contains electron-exchange terms not present in the SPM cross section. These Pauli exchange terms (PET's) become more numerous as the specified number of occupancies or nonoccupancies is increased. Each PET contains ${ }^{6}$ a product of off-diagonal matrix elements of the "singleelectron, transition-density" matrix, $a^{(2)},{ }^{3}$ defined by

$$
a_{k k^{\prime}}^{(2)}(b)=\sum_{h}^{\mathrm{occ}} a_{k h}^{*}(b) a_{k^{\prime} h}(b)=a_{k^{\prime} k}^{(2) *}(b) .
$$

These are complex-valued coherent superpositions of bilinear products of amplitudes for different single-electron transitions. The element $a_{k k^{\prime}}^{(2)}$ can be comparable to or small compared to $\left(\rho^{k} \rho^{k^{\prime}}\right)^{1 / 2}$, depending on whether the interference of the terms in (1.6) is constructive or destructive. Moreover, for any inclusive process, the sum of PET's can also be large or small compared to the average magnitude of one PET, depending on whether the interference of PET's is constructive or destructive. Thus the PET's provide a mechanism for the coherent enhancement or suppression of otherwise (in the SPM) incoherent, independent-electron transitions.

An example of a hyperinclusive doubles measurement is the experiment first performed by MacDonald ${ }^{7}$ in which one detects an electron capture from the target (by measuring the final ionic charge of the projectile) in coincidence with a hole in the $K$ shell of the target (detected from its decay by the emission of a $K \times$ ray). We have found $^{8}$ that the PET's provide a significant enhancement of this process at impact speeds below the "matching" speed (i.e., the average speed of a $K$-shell electron in the target) and that this enhancement grows with the charge of the projectile.

We were stimulated by our investigation of the PET enhancement of the capture-and-vacancy production process to ask whether striking enhancements or suppressions resulting from PET's might occur for inclusive $p$-fold coincidences with $p$ greater than two. The process most accessible experimentally is ion-induced multiple-vacancy production. The multiple-vacancy configurations $K L^{v}$ can be detected as satellites of the $K \alpha$ x-ray or Auger lines. Such measurements have been made frequently ${ }^{2}$ since the first high-resolution satellite work in $1972 .{ }^{9}$

The $K$ x-ray satellites, as observed with moderate resolution, usually are characterized by the number of $L$-shell vacancies, but not by the number of $M-, N-, \ldots$ shell vacancies nor by the multiplet structure. These latter differences can be detected with a spectrometer of very high resolution, but we shall assume that the measurements under discussion are inclusive with respect to everything except the number of $L$-shell vacancies or the numbers of $2 s$ and $2 p$ vacancies. We need to distinguish a subset of hyperinclusive probabilities which we shall call numberexclusive hyperinclusive (NEHI) probabilities. If an initially full shell, $S$, contains $N$ spin orbitals, a NEHI probability for this shell will specify a precise number of vacancies $v$ and the complementary precise number of occupancies $N-v$. We shall denote it by $P_{S^{v}}^{S^{N-v}}$. Most hyperinclusive quantities are not exclusive with respect to the numbers of occupancies and nonoccupancies. For exam- 
ple, $P_{S^{v}}$ and $P^{S^{n}}$ refer to processes with at least $v$ nonoccupancies and at least $n$ occupancies, respectively, in the shell $S$. Only when the specified number of occupancies in a shell plus the specified number of nonoccupancies in the shell equals the total number of single-particle states in the full shell is a hyperinclusive probability a NEHI one. $P_{S^{v}}^{S^{N-v-1}}$ is not a NEHI probability. It includes the possibility of $N-v-1$ occupancies and $v+1$ nonoccupancies and also the possibility of $N-v$ occupancies and $v$ nonoccupancies.

The main purpose of this paper is to present a derivation of general formulas ${ }^{3,10}$ for NEHI probabilities in terms of simpler hyperinclusive expected values for at least $v$ vacancies, $Q_{S^{v}}$, or for at least $n$ occupancies, $Q^{S^{n}}$, which are sums of determinants. The use of the $Q_{S^{v}}$ will be referred to as the "vacancy formalism," and the use of the $Q^{S^{n}}$ gives the "occupancy formalism." Numerical calculations have been made with both formalisms. The results are the same. This provides an important check on the validity of both sets of formulas. The essential nature of these formulas is contained in the result for a single shell. In the vacancy formalism, with $Q_{S^{v}}$ defined by

$$
Q_{S^{v}}(N) \equiv \sum_{\lambda_{1}<\cdots<\lambda_{v}} \rho_{\lambda_{1}, \ldots, \lambda_{v}} \leq\left(\begin{array}{c}
N \\
v
\end{array}\right),
$$

where each spin orbital $\lambda_{i}$ belongs to $S$, the NEHI probabilities are given by ${ }^{3}$

$$
\begin{aligned}
P_{S^{v}}^{S^{N-v}}= & Q_{S^{v}}-\left(\begin{array}{c}
v+1 \\
1
\end{array}\right) Q_{S^{v+1}}+\left(\begin{array}{c}
v+2 \\
2
\end{array}\right) Q_{S^{v+2}}+\cdots \\
& +(-1)^{N-v}\left[\begin{array}{c}
N \\
N-v
\end{array}\right) Q_{S^{N}}
\end{aligned}
$$

The first term contains the possibility of more than $v$ vacancies; the second term then removes the unwanted $v+1$ vacancies in $Q_{S}$; the third term removes the unwanted $v+2$ vacancies in

$$
Q_{S^{v}}-\left(\begin{array}{c}
v+1 \\
1
\end{array}\right) Q_{S^{v+1}},
$$

etc. A general inversion theorem which leads to this result is given in an Appendix.

The present paper employs only one basis of singleelectron spin orbitals. The HF field which generates this basis is static, although the generalization of the multiple-vacancy formalism to time-dependent HartreeFock fields appears straightforward. The present treatment therefore neglects shake-off and shake-up contributions to vacancy production and neglects the difference between the $L$-shell removal energies of satellite and hypersatellite configurations. Preliminary discussions of these effects have been given, ${ }^{10-12}$ and a more detailed account is being completed. ${ }^{5}$

In Sec. II we give a brief review of the IFPM expressions for the probabilities of particular inclusive processes. $^{6,8}$ Section III defines hyperinclusive quantities ${ }^{3}$ and discusses their experimental significance in the case of a single shell. The expected values for at least $v$ vacancies are expressed in terms of the number-exclusive probabilities. Then these relations are inverted to provide explicit expressions for the NEHI probabilities as linear combinations of determinants. It is shown that these IFPM formulas reduce to those of the SPM when the PET's are set equal to zero. Section IV provides the generalization to two and three shells in the cases of the $K$ and $L$ shells and of the $K, 2 s$, and $2 p$ shells, respectively. We discuss the approach of the IFPM vacancy distribution to the SPM (binomial) distribution in Sec. V. This occurs generally when the mean vacancy probability per electron $\bar{p}$ is small and can, and does, ${ }^{10,12}$ occur also for large $\bar{p}$ when the single-particle amplitudes tend to have random phases. The concepts introduced and a general inversion theorem proved in an Appendix permit generalization of the multiple-vacancy expressions to other cases.

\section{IFPM PARTICULAR INCLUSIVE PROBABILITIES}

IFPM formulas for particular inclusive processes have been derived, discussed, and applied in a few previous papers. ${ }^{6,8}$ Because it is essential to have these results in mind in order to understand the present paper, we summarize them briefly.

The quantity $\left\langle k_{1}, \ldots, k_{F}|A(b)| h_{1}, \ldots, h_{F}\right\rangle$ will denote the amplitude for the transition, in an ion-atom collision with impact parameter $b$, from the normalized Slater determinant with $F$ target-centered spin-orbitals $h=h_{1}, \ldots, h_{F}$, see Eq. (1.1), to one with $F$ spin orbitals $k=k_{1}, \ldots, k_{F}$. In terms of the single-electron transition amplitudes, $a_{k h}(b)$,

$$
\left\langle k_{1}, \ldots, k_{F}|A(b)| h_{1}, \ldots, h_{F}\right\rangle=\operatorname{det}\left[a_{k h}\right],
$$

where $k$ ranges over $k_{1}, \ldots, k_{F}$ and $h$ over $h_{1}, \ldots, h_{F}$.

For the purposes of the present paper, it is irrelevant how the $a_{j \alpha}$ 's are obtained. In our own work ${ }^{8}$

$$
a_{j \alpha}(b)=\lim _{t \rightarrow \infty}\left\langle\chi_{j}(b, t) \mid \psi_{\alpha}^{(+)}(b, t)\right\rangle,
$$

where $\chi_{j}$ is a stationary single-electron state of the unperturbed system with no interaction between the projectile and target and $\psi_{\alpha}^{(+)}$is a perturbed spin orbital subject to the initial condition that

$$
\psi_{\alpha}^{(+)}(b, t) \rightarrow \chi_{\alpha}(b,-\infty) \text { as } t \rightarrow-\infty .
$$

It satisfies the time-dependent Schrödinger equation

$$
\left[i \hbar \frac{\partial}{\partial t}+\frac{\hbar^{2}}{2 m} \nabla_{r}^{2}-U_{\mathrm{HF}}(\overrightarrow{\mathrm{r}})-V(\overrightarrow{\mathrm{r}} ; b, t)\right] \psi_{\alpha}(\overrightarrow{\mathrm{r}}, t)=0,
$$

where $U_{\mathrm{HF}}$ is the static Hartree-Fock potential of the target and $V(\overrightarrow{\mathrm{r}} ; b, t)$ is the perturbing interaction provided by the projectile while moving on a classical trajectory that is assumed to be undisturbed by any electronic excitation. The formalism of this paper is unchanged if $U_{\mathrm{HF}}(\overrightarrow{\mathrm{r}})$ is replaced by a time-dependent potential such as that of the time-dependent Hartree-Fock theory. However, because in this paper we use only a single basis of unperturbed states $\chi_{j}(\overrightarrow{\mathbf{r}} ; b, t)$ generated by a static potential, the present 
theory does not take into account shake-off and shake-up processes, which are important at high impact velocities and for light targets and are more significant for hypersatellites than for satellites.

The probability for the exclusive reaction $\left(h_{1}, \ldots\right.$, $\left.h_{F} \rightarrow k_{1}, \ldots, k_{F}\right)$ is

$$
\begin{aligned}
\rho^{k_{1}, \ldots, k_{F}}\left(h_{1}, \ldots, h_{F} ; b\right) & \\
& =\left|\left\langle k_{1}, \ldots, k_{F}|A(b)| h_{1}, \ldots, h_{F}\right\rangle\right|^{2} .
\end{aligned}
$$

Particular inclusive probabilities in which only final occupancies are specified are defined here by

$\rho^{k_{1}, \ldots, k_{m}}(F)=\sum_{k_{m+1}<\cdots<k_{F}} \rho^{k_{1}, \ldots, k_{F}}, \quad m=1, \ldots, F-1$,

where we have suppressed the dependence on the initial spin orbitals and on the impact parameter. The prime on the sum means that $k_{m+1}, \ldots, k_{F}$ must not equal any of the specified spin orbitals $k_{1}, \ldots, k_{m}$. Because of the antisymmetry of the Slater determinant $\left\langle k_{1}, \ldots, k_{F}\right|$, this restriction on the sum may be dropped. Because of the completeness of the basis of spin orbitals, one finds ${ }^{6}$

$$
\rho^{k_{1}, . ., k_{m}}(F)=\operatorname{det}\left[a_{k_{i}, k_{j}}^{(2)}\right],
$$

the determinant of an $m \times m$ matrix $(i, j=1, \ldots, m)$. Here $a^{(2)}$ is as defined in Eq. (1.6), with $h=h_{1}, \ldots, h_{F}$. Similarly, particular inclusive probabilities in which only final nonoccupancies are specified are defined for any $m=1,2, \ldots$ by

$$
\begin{aligned}
\rho_{v_{1}}, \ldots, v_{m} & (F) \equiv \sum_{k_{1}<\cdots<k_{F}} \rho^{k_{1}, \ldots, k_{F}}, \\
& k_{i} \neq v_{1}, \ldots, v_{m},
\end{aligned}
$$

where $v_{i} \neq v_{j}$ for $i \neq j$ and $i, j=1,2, \ldots, m$. We find

$$
\rho_{v_{1}, \ldots, v_{m}}(F)=\operatorname{det}\left[\delta_{i j}-a_{k_{i}, k_{j}}^{(2)}\right], \quad i, j=1, \ldots, m \text {. }
$$

For $m=1$ these expressions reduce to Eqs. (1.4) and (1.5). For $m=2$ Eq. (2.6) gives for $k_{1} \neq k_{2}$

$$
\rho_{k_{1}, k_{2}}=\rho_{k_{1}} \rho_{k_{2}}-\left|a_{k_{1} k_{2}}^{(2)}\right|^{2} \leq \rho_{k_{1}} \rho_{k_{2}},
$$

where the PET always gives a suppression of the SPM probability, $\rho_{k_{1}} \rho_{k_{2}}$. Similarly,

$$
\rho^{k_{1}, k_{2}}=\rho^{k_{1}} \rho^{k_{2}}-\left|a_{k_{1} k_{2}}^{(2)}\right|^{2} \leq \rho^{k_{1}} \rho^{k_{2}} .
$$

By writing out Eq. (2.7b) in detail,

$$
\begin{aligned}
\rho^{k_{1}, k_{2}}= & \sum_{h}^{\mathrm{occ}}\left|a_{k_{1} h}\right|^{2} \sum_{h^{\prime}}^{\mathrm{occ}}\left|a_{k_{2} h^{\prime}}\right|^{2} \\
& -\sum_{h}^{\mathrm{occ}} a_{k_{1} h}^{*} a_{k_{2} h} \sum_{h^{\prime}}^{\mathrm{occ}} a_{k_{1} h^{\prime}} a_{k_{2} h^{\prime}}^{*}
\end{aligned}
$$

we see that the PET corrections to the SPM result are of two kinds. First, the terms with $h^{\prime}=h$ cancel similar terms in $\rho^{k_{1}} \rho^{k_{2}}$. This cancellation eliminates unphysical terms of the form $\left|a_{k_{1} h}\right|^{2}\left|a_{k_{2} h}\right|^{2}$ which would correspond to an electron initially in spin orbital $h$ making a transition both to $k_{1}$ and to $k_{2}$. We refer to this elimination as a single-electron Pauli correction. It is somewhat analogous to the elimination of the Hartree interaction of an electron with itself by the corresponding Fock term in the HF theory. Second, there are coherent electronexchange terms

$$
-\sum_{h}^{\mathrm{occ}} \sum_{h^{\prime}}^{\mathrm{occ}} a_{(\neq h)}^{*} a_{k_{1} h} a_{k_{1} h^{\prime}} a_{k_{2} h^{\prime}}^{*} a_{k_{2} h} .
$$

Particular inclusive probabilities in which some occupancies and some nonoccupancies are specified are given by sums of determinants. One can obtain these from Eq. (2.4) or Eq. (2.6) by repeated use of a raising and lowering relation, namely for any $m \leq F-1$

$$
\rho_{v_{1}, \ldots, v_{n}}^{k_{1}, \ldots, k_{m}}(F)=\rho_{v_{1}, \ldots, v_{n}, k}^{k_{1}, \ldots, k_{m}}(F)+\rho_{v_{1}, \ldots, v_{n}}^{k_{1}, \ldots, k_{m}, k}(F) .
$$

Thus for $(m=1, n=0)$ or $(m=0, n=1)$, by using Eq. (1.5), we obtain

$$
\rho_{k_{2}}^{k_{1}}(N)=\rho^{k_{1}} \rho_{k_{2}}+\left|a_{k_{1} k_{2}}^{(2)}\right|^{2} \geq \rho^{k_{1}} \rho_{k_{2}}, \quad k_{1} \neq k_{2}
$$

where the PET always gives an enhancement over the uncorrelated SPM value, $\rho^{k_{1}} \rho_{k_{2}}$. The SPM contribution contains single-electron terms.

$$
-\sum_{h}^{o c c}\left|a_{k_{1} h}\right|^{2}\left|a_{k_{2} h}\right|^{2},
$$

which correspond to the subtraction of quantities in which an electron initially in $h$ contributes both to the occupancy of $k_{1}$ and of $k_{2}$. These spurious terms are cancelled by the diagonal $\left(h^{\prime}=h\right)$, i.e., single-electron, terms in $\left|a_{k_{1} k_{2}}^{(2)}\right|^{2}$. Thus, after the cancellations, we can write $\rho_{k_{2}}^{k_{1}}$ as the sum of two terms, each of which involves electron-electron correlations:

$$
\rho_{k_{2}}^{k_{1}}={ }^{\operatorname{dir}} \rho_{k_{2}}^{k_{1}}+{ }^{\mathrm{ex}} \rho_{k_{2}}^{k_{1}},
$$

where the direct term is

$$
{ }^{\operatorname{dir}} \rho_{k_{2}}^{k_{1}}=\sum_{h}^{\mathrm{occ}}\left|a_{k_{1} h}\right|^{2}\left(1-\sum_{h^{\prime}(\neq h)}^{\mathrm{occ}}\left|a_{k_{2} h^{\prime}}\right|^{2}\right)
$$

and the exchange term is

$$
{ }^{\operatorname{ex}} \rho_{k_{2}}^{k_{1}}=\sum_{h}^{\mathrm{occ}} \sum_{h^{\prime}(\neq h)}^{\mathrm{occ},} a_{k_{1} h}^{*} a_{k_{2} h^{\prime}}^{*} a_{k_{1} h^{\prime}} a_{k_{2} h} .
$$

Detailed coupled-channel calculations of $\rho_{h_{2}}^{p_{1}}$, where $p_{1}$ is an electron-capture state, have been made earlier ${ }^{8}$ and compared with data from coincidence experiments. ${ }^{7}$ The PET enhancement shows up more strongly for larger projectile charge and lower impact speed.

The description of multiple-vacancy production, detectable in X-ray and Auger satellites, involves particular inclusive probabilities $\rho_{v_{1}}, \ldots, v_{m}$, Eq. (2.6). However, because the particular vacancy states with the same number of $K$-shell and $L$-shell vacancies are not distinguished 
from one another at the moderate resolution of the experiments, one must sum over the various ways of producing the same number of vacancies. We call such sums of particular inclusive probabilities hyperinclusive ${ }^{3}$ quantities. They are defined and evaluated in the next two sections.

\section{HYPERINCLUSIVE PROBABILITIES AND EXPECTED VALUES FOR A SINGLE SHELL}

\section{A. Shell with only two spin orbitals}

We introduce hyperinclusive quantities ${ }^{3}$ by considering. the simplest case, shells with only two single-particle states, i.e., with $j=\frac{1}{2}$, namely the shells $S=n s_{1 / 2}$ and $n p_{1 / 2}$. There are four exclusive probabilities, $\rho^{12}, \rho_{2}^{1}, \rho_{1}^{2}$, and $\rho_{12}$, and four particular inclusive probabilities,

$$
\begin{array}{ll}
\rho_{1}=\rho_{1}^{2}+\rho_{12}, & \rho_{2}=\rho_{2}^{1}+\rho_{12}, \\
\rho^{1}=\rho_{2}^{1}+\rho^{12}, & \rho^{2}=\rho_{1}^{2}+\rho^{12} .
\end{array}
$$

Hyperinclusive quantities refer to shells rather than to particular spin orbitals. The ones we are primarily interested in for describing multiple-vacancy production specify precisely the number of vacancies in the shell in question, and also its complement, the number of occupancies in the shell. We call these number-exclusive hyperinclusive (NEHI) probabilities. They are inclusive with respect to angular momentum couplings in the shell in question and with respect to all electrons or holes in other shells. In the $j=\frac{1}{2}$ case the NEHI probabilities are

$$
P_{S^{0}}^{S^{2}} \equiv P^{S^{2}} \equiv \rho^{1,2}, \quad P_{S^{2}}^{S^{0}} \equiv P_{S^{2}} \equiv \rho_{1,2}
$$

which are single determinants, and

$$
\begin{aligned}
P_{S^{1}}^{S^{1}} \equiv P_{S}^{S} \equiv \rho_{2}^{1}+\rho_{1}^{2} & =\left(\rho^{1}+\rho^{2}\right)-2 \rho^{1,2} \\
& =\left(\rho_{1}+\rho_{2}\right)-2 \rho_{1,2}
\end{aligned}
$$

which is a sum of three determinants. Other, nonnumber-exclusive, hyperinclusive probabilities for shell $S$ may be defined by analogy with Eqs. (3.1):

$$
\begin{aligned}
& P^{S} \equiv P_{S}^{S}+P^{S^{2}}=\rho^{1}+\rho^{2}-\rho^{1,2}=\rho_{2}^{1}+\rho_{1}^{2}+\rho^{1,2}, \\
& P_{S} \equiv P_{S}^{S}+P_{S^{2}}=\rho_{1}+\rho_{2}-\rho_{1,2}=\rho_{2}^{1}+\rho_{1}^{2}+\rho_{1,2} .
\end{aligned}
$$

$P^{S}$ and $P_{S}$ are the probabilities that at least one spin orbital of the $S$ shell is occupied or not occupied, respectively. They satisfy the desired relations

$$
P^{S}+P_{S^{2}}^{S^{0}}=1=P_{S}+P_{S^{0}}^{S^{2}}
$$

because from Eq. (2.9)

$$
\left.\begin{array}{l}
P^{S}+P_{S^{2}}^{S^{0}} \\
P_{S}+P_{S^{0}}^{S^{2}}
\end{array}\right\}=\rho_{2}^{1}+\rho_{1}^{2}+\rho^{1,2}+\rho_{1,2}=\left\{\begin{array}{c}
\rho^{1}+\rho_{1} \\
\rho^{2}+\rho_{2}
\end{array}\right\}=1 .
$$

Because of the existence of the general formulas (2.4) and (2.6), it will be advantageous to work with quantities in which all the specified states are occupied or are not occupied. We introduce the hyperinclusive "expected values"

$$
\begin{aligned}
& Q^{S} \equiv \rho^{1}+\rho^{2}=\rho_{2}^{1}+\rho_{1}^{2}+2 \rho^{1,2}, \\
& Q_{S} \equiv \rho_{1}+\rho_{2}=\rho_{1}^{2}+\rho_{2}^{1}+2 \rho_{1,2}, \\
& Q^{S^{2}}=\rho^{1,2}, Q_{S^{2}}=\rho_{1,2} .
\end{aligned}
$$

From (3.4) we see that

$$
\begin{array}{ll}
Q^{S}=P^{S}+P^{S^{2}}=P_{S}^{S}+2 P_{S^{0}}^{S^{2}}, & Q^{S^{2}}=P_{S^{0}}^{S^{2}}, \\
Q_{S}=P_{S}+P_{S^{2}}=P_{S}^{S}+2 P_{S^{2}}^{S^{0}}, & Q_{S^{2}}=P_{S^{2}}^{S^{0}} .
\end{array}
$$

If $\rho_{1}=\rho_{2} \rightarrow 1$, then $Q_{S} \rightarrow 2$ and, if $a_{1,2}^{(2)}=0, \rho_{1,2} \rightarrow 1$ so that $P_{S} \rightarrow 1$ and $P_{S}^{S} \rightarrow P_{S^{0}}^{S^{2}} \rightarrow 0$. If $\rho_{1}=\rho_{2}=\rho^{1}=\rho^{2}=\frac{1}{2}$, then $Q_{S}=1$ and if $a_{12}^{(2)}=0, \rho_{1,2}=\frac{1}{4}$ so $P_{S}=\frac{3}{4}=P^{S}$ and $P_{S}^{S}=\frac{1}{2}$.

The main result we seek is the expression for each NEHI probability in terms of the $Q$ 's. For $N=2$, the result is trivial:

$$
\begin{aligned}
& P_{S^{2}}^{S^{0}}=Q_{S^{2}}, \quad P_{S}^{S}=Q_{S}-2 Q_{S^{2}}, \\
& P_{S^{0}}^{S^{2}}=Q^{S^{2}}, \quad P_{S}^{S}=Q^{S}-2 Q^{S^{2}} .
\end{aligned}
$$

We shall refer to expressions in which the only $Q$ 's which appear have subscripts, e.g., Eq. (3.8), as being in the vacancy formalism, and to those like Eq. (3.9) as being in the occupancy formalism. For completeness, we still need $P_{S^{0}}^{S^{2}}$ in the vacancy formalism and $P_{S^{2}}^{S^{0}}$ in the occupancy formalism. From the raising and lowering relation, Eq. (2.9), we have

$$
P_{S^{0}}^{S^{2}}=\rho^{1,2}=1-\left(\rho_{1}+\rho_{2}\right)+\rho_{1,2}=Q_{S^{0}}-Q_{S}+Q_{S^{2}}
$$

and

$$
P_{S^{2}}^{S^{0}}=\rho_{1,2}=1-\left(\rho^{1}+\rho^{2}\right)+\rho^{1,2}=Q^{S^{0}}-Q^{S}+Q^{S^{2}}
$$

where we have defined

$$
Q_{S^{0}} \equiv 1 \equiv Q^{S^{0}} .
$$

Let us now consider the physical significance of the hyperinclusive quantities. For definiteness we shall assume the shell $S$ is the $K$ shell. Moreover, we shall assume that the fluorescence yields (i.e., the branching ratios for decay by $\mathrm{x}$-ray emission) are known or that the experiment can detect both $\mathrm{x}$ rays and Auger electrons. Both $\rho_{2}^{1}$ and $\rho_{1}^{2}$ represent a single $K$ vacancy, which leads to a single decay product. But $\rho_{1,2}$ represents two $K$ vacancies, which lead to two decay products. We shall assume these decay products have distinguishably different energies, the first in the "hypersatellite group" and the second in the "satellite group." For some ions these groups overlap, but we shall ignore that possibility. From (3.2) and (3.6) we see that $Q_{K^{2}}=P_{K^{2}}=\rho_{1,2}$ is the probability for two $K$ vacancies, which corresponds to the detection of a hypersatellite decay. The measurement requires sufficiently high energy resolution to distinguish it from a satellite decay. From 
(3.4) we find that $P_{K}$ corresponds to the detection of all satellite decays. Of the two decays from the double vacancy described by $\rho_{1,2}$, only one decay is to be counted. Again this measurement requires high energy resolution. From (3.3) $P_{K}^{K}$ represents satellite configurations and corresponds to measurement of satellite decays not accompanied by hypersatellite decays. From (3.4) $P_{K}^{K}$ is just the difference between $P_{K}$ and $P_{K^{2}}$, or, from (3.7), is the difference between $Q_{K}$ and $2 P_{K^{2}}$. We see from (3.6) that $Q_{K}$ contains $2 \rho_{1,2}$ so that both decays from the double $K$ vacancy configurations are to be counted. Thus $Q_{K}$ corresponds to an experiment, which could be of lower resolution, in which satellite and hypersatellite decays are not distinguished and all decays are counted. Alternatively, $P_{K}$ corresponds to the detection of all prompt decays, $Q_{K}$ corresponds to the detection of all decays, including second decays, and $Q_{K^{2}}=P_{K^{2}}$ corresponds to the detection of near coincidences between two decays occurring roughly within the lifetime of a single $K$ vacancy. In summary, the measurement of the NEHI $\left(P_{K^{2}}\right.$ and $\left.P_{K}^{K}\right)$ and nonNEHI $\left(P_{K}\right)$ probabilities requires high resolution in energy or time, whereas the expected value which is not a probability $\left(Q_{K}\right)$ corresponds to a measurement of low resolution.

\section{B. Shell with $N$ spin orbitals}

To simplify the notation, we drop the reference to the shell $S$ and write $P_{S^{v}}^{S^{N-v}}=P_{v}^{N-v}, Q_{S^{v}}=Q_{v}$, etc. We define the NEHI probabilities by

$$
\begin{aligned}
& P_{v}^{N-v}=\sum_{\lambda_{1}<\cdots<\lambda_{v}} \sum_{\lambda_{v+1}<\cdots<\lambda_{N}} \rho_{\lambda_{1}, \ldots, \lambda_{v}}^{\lambda_{v+1}, \ldots, \lambda_{N}} \\
& =\frac{1}{v !(N-v) !} \sum_{\lambda_{1}, \ldots, \lambda_{N}} \rho_{\lambda_{1}, \ldots, \lambda_{v}}^{\lambda_{\lambda_{1}}, \ldots, \lambda_{N}},
\end{aligned}
$$

where each $\lambda_{i}$ belongs to $S$. Terms in which two or more $\lambda$ 's are the same vanish because of the exclusion principle, i.e., because the $\rho$ 's are obtained from antisymmetric wave functions. The hyperinclusive expected values are defined $^{3}$ for $1 \leq v$ or $n \leq N$ by

$$
Q_{v} \equiv \sum_{\lambda_{1}<\cdots<\lambda_{v}} \rho_{\lambda_{1}}, \ldots, \lambda_{v}=\frac{1}{v !} \sum_{\lambda_{1}, \ldots, \lambda_{v}} \rho_{\lambda_{1}}, \ldots, \lambda_{v}
$$

and

$$
Q^{n} \equiv \sum_{\lambda_{1}<\cdots<\lambda_{n}} \rho^{\lambda_{1}, \ldots, \lambda_{n}}=\frac{1}{n !} \sum_{\lambda_{1}, \ldots, \lambda_{n}} \rho^{\lambda_{1}, \ldots, \lambda_{n}},
$$

together with Eq. (3.12) for $v$ or $n$ equal to zero. We shall discuss the vacancy formalism; the results for the occupancy formalism can be obtained in a similar way. Let us denote the spin orbitals in $S$ by $1,2, \ldots, N$. First we need to express the $Q_{v}$ 's in terms of the NEHI probabilities. Then these relations will be inverted. We begin with $Q_{N}$ and work down to $Q_{1}$. We have

$$
Q_{N}=\rho_{1,2, \ldots, N}=P_{N}^{0},
$$

a single determinant. For each of the $N$ terms of $Q_{N-1}$ we can write

$$
\rho_{\lambda_{1}, \ldots, \lambda_{N-1}}=\rho_{\lambda_{1}, \ldots, \lambda_{N-1}}^{\lambda_{N}}+\rho_{1,2, \ldots, N},
$$

so

$$
Q_{N-1}=P_{N-1}^{1}+N P_{N} \text {. }
$$

The general term in $Q_{N-2}$ can be written

$$
\begin{aligned}
& \rho_{\lambda_{1}, \ldots, \lambda_{N-2}}=\frac{1}{2 !} \sum_{\lambda_{N-1}, \lambda_{N}} \begin{array}{c}
\lambda_{\lambda_{1-1}, \lambda_{N}, \ldots, \lambda_{N-2}}+2 \rho_{\lambda_{1}}^{\lambda_{N}} \ldots, \lambda_{N-1} \\
\rho^{2}
\end{array} \\
& \left.+\rho_{\lambda_{1}, \ldots, \lambda_{N}}\right),
\end{aligned}
$$

so

$$
\begin{aligned}
Q_{N-2} & =P_{N-2}^{2}+\frac{(N-1) !}{(N-2) !} P_{N-1}^{1}+\frac{N !}{(N-2) ! 2 !} P_{N}^{0} \\
& =\left(\begin{array}{l}
N-2 \\
N-2
\end{array}\right) P_{N-2}^{2}+\left[\begin{array}{l}
N-1 \\
N-2
\end{array}\right) P_{N-1}^{1}+\left[\begin{array}{c}
N \\
N-2
\end{array}\right) P_{N}^{0} .
\end{aligned}
$$

For $Q_{N-3}$ we have

$$
\begin{aligned}
& \rho_{\lambda_{1}, \ldots, \lambda_{N-3}}=\frac{1}{3 !} \sum_{\lambda_{N-2}, \ldots, \lambda_{N}} \begin{array}{c}
\left(\rho_{\lambda_{1}, \ldots, \lambda_{N-3}}^{\lambda_{N-1}, \lambda_{N-1}, \lambda_{N}}+3 \lambda_{\lambda_{1}, \ldots, \lambda_{N-2}}^{\lambda_{N-1}, \lambda_{N}}\right. \\
\rho_{\lambda_{1}}, \ldots, \lambda_{N-2}
\end{array} \\
& \left.+3 \rho_{\lambda_{1}, \ldots, \lambda_{N-1}}^{\lambda_{N}}+\rho_{\lambda_{1}, \ldots, \lambda_{N}}\right) .
\end{aligned}
$$

Hence

$$
\begin{aligned}
Q_{N-3}= & {\left[\begin{array}{l}
N-3 \\
N-3
\end{array}\right) P_{N-3}^{3}+\left[\begin{array}{l}
N-2 \\
N-3
\end{array}\right) P_{N-2}^{2} } \\
& +\left[\begin{array}{l}
N-1 \\
N-3
\end{array}\right) P_{N-1}^{1}+\left[\begin{array}{c}
N \\
N-3
\end{array}\right) P_{N}^{0} .
\end{aligned}
$$

We find that, in general, for a set $S$ with $N$ spin orbitals,

$$
Q_{N-n}(N)=\sum_{j=N-n}^{N}\left[\begin{array}{c}
j \\
N-n
\end{array}\right) P_{j}^{N-j}
$$

or, since $\left(\begin{array}{l}j \\ v\end{array}\right)=0$ for $j<v$,

$$
Q_{v}(N)=\sum_{j=0}^{N}\left(\begin{array}{l}
j \\
v
\end{array}\right) P_{j}^{N-j} .
$$

Similarly,

$$
Q^{n}(N)=\sum_{j=0}^{N}\left(\begin{array}{l}
j \\
n
\end{array}\right) P_{N-j}^{j} .
$$

These results contrast with those for the hyperinclusive probabilities

$$
P_{v}=\sum_{j=v}^{N} P_{j}^{N-j}, \quad P^{n}=\sum_{j=n}^{N} P_{N-j}^{\dot{j}} .
$$

For $v=0$ Eq. (3.15) confirms the normalization of the distribution,

$$
1=\sum_{j=0}^{N} P_{j}^{N-j},
$$

and for $v=1$ and 2 it implies ${ }^{13,10}$ for the inclusive "singles" and "doubles" cross sections in which all decay products are detected 


$$
\begin{aligned}
& \sigma_{S}(Q)=\sum_{v=1}^{N} v \sigma_{S^{v}}^{S^{N-v}}, \\
& \sigma_{S^{2}}(Q)=\sum_{v=2}^{N}\left[\begin{array}{l}
v \\
2
\end{array}\right] \sigma_{S^{v}}^{S^{N-v}} .
\end{aligned}
$$

On the other hand, if only the last decay product is observed, one has

$$
\sigma_{S}(P)=\sum_{v=1}^{N} \sigma_{S^{v}}^{S^{N-v}}
$$

and, if only the last two decay products are observed,

$$
\sigma_{S^{2}}(P)=\sum_{v=2}^{N} \sigma_{S^{v}}^{S^{N-v}}
$$

The inversion of (3.15) can be done successively, starting with $v=N$. One has

$$
P_{N}^{0}=Q_{N}, \quad P_{N-1}=Q_{N-1}-\left[\begin{array}{c}
N \\
N-1
\end{array}\right) Q_{N},
$$

and

$$
P_{N-2}^{2}=Q_{N-2}-\left(\begin{array}{c}
N-1 \\
N-2
\end{array}\right) Q_{N-1}+\left(\begin{array}{c}
N \\
N-2
\end{array}\right) Q_{N}
$$

Then

$$
\begin{aligned}
P_{N-3}^{3}= & Q_{N-3}-\left[\begin{array}{l}
N-2 \\
N-3
\end{array}\right] Q_{N-2}+\left[\left(\begin{array}{l}
N-2 \\
N-3
\end{array}\right]\left[\begin{array}{l}
N-1 \\
N-2
\end{array}\right]-\left(\begin{array}{l}
N-1 \\
N-3
\end{array}\right)\right] Q_{N-1} \\
& -\left[\left(\begin{array}{l}
N-2 \\
N-3
\end{array}\right]\left[\begin{array}{c}
N \\
N-2
\end{array}\right)-\left[\begin{array}{l}
N-1 \\
N-3
\end{array}\right]\left[\begin{array}{c}
N \\
N-1
\end{array}\right)+\left[\begin{array}{c}
N \\
N-3
\end{array}\right]\right] Q_{N} \\
= & Q_{N-3}-\left(\begin{array}{l}
N-2 \\
N-3
\end{array}\right) Q_{N-2}+\left(\begin{array}{l}
N-1 \\
N-3
\end{array}\right) Q_{N-1}-\left(\begin{array}{c}
N \\
N-3
\end{array}\right) Q_{N} .
\end{aligned}
$$

We find that the general NEHI probability is given by

$$
\begin{aligned}
P_{v}^{N-v} & =\sum_{j=0}^{N-v}(-1)^{j}\left[\begin{array}{c}
v+j \\
v
\end{array}\right] Q_{v+j} \\
& =\sum_{i=0}^{N}(-1)^{i+v}\left(\begin{array}{l}
i \\
v
\end{array}\right] Q_{i} .
\end{aligned}
$$

A noniterative proof of the inversion formula is given in the Appendix. In the occupancy formalism we have

$$
\begin{aligned}
P_{N-n}^{n} & =\sum_{j=0}^{N-n}(-1)^{j}\left(\begin{array}{c}
n+j \\
j
\end{array}\right) Q^{n+j} \\
& =\sum_{i=0}^{N}(-1)^{i+n}\left(\begin{array}{l}
i \\
n
\end{array}\right) Q^{i}
\end{aligned}
$$

or

$$
\begin{aligned}
P_{v}^{N-v} & =\sum_{j=0}^{v}(-1)^{j}\left[\begin{array}{c}
N-v+j \\
j
\end{array}\right) Q^{N-v+j} \\
& =\sum_{i=0}^{N}(-1)^{i+N-v}\left[\begin{array}{c}
i \\
N-v
\end{array}\right) Q^{i}
\end{aligned}
$$

The occupancy form has fewer terms when $v<N / 2$, and the vacancy form is more convenient when $v>N / 2$.

We now show that these IFPM formulas reduce to a binomial distribution when the off-diagonal elements $a_{i j}^{(2)}$, $i \neq j$, in the determinants which give the particular inclusive probabilities [Eqs. (2.4) and (2.6)], are set equal to zero. ${ }^{3}$ In this limit the product of independent inclusive probabilities is recovered:

$$
\begin{aligned}
& \rho_{\lambda_{1}, \ldots, \lambda_{v}}=\prod_{i=1}^{v} \rho_{\lambda_{i}} \\
& \rho^{\lambda_{1}, \ldots, \lambda_{n}}=\prod_{i=1}^{n} \rho^{\lambda_{i}} \\
& (S P M) .
\end{aligned}
$$

If one also makes the "average hole" assumption ${ }^{3}$ that

$$
1-\rho^{\lambda_{i}}(b) \equiv \rho_{\lambda_{i}}(b) \rightarrow p(b),
$$

then at a given value of the impact parameter

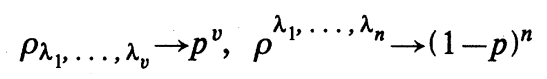

and

$$
Q_{v} \rightarrow\left(\begin{array}{c}
N \\
v
\end{array}\right) p^{v}, \quad Q^{n} \rightarrow\left(\begin{array}{l}
N \\
n
\end{array}\right)(1-p)^{n}
$$

By Eq. (3.21)

$$
\begin{aligned}
P_{v}^{N-v} & \rightarrow \sum_{j=0}^{N-v}(-1)^{j}\left[\begin{array}{c}
v+j \\
j
\end{array}\right]\left[\begin{array}{c}
N \\
v+j
\end{array}\right] p^{v+j} \\
& =\left[\begin{array}{c}
N \\
n
\end{array}\right) p^{v} \sum_{j=0}^{N-v}(-1)^{j}\left(\begin{array}{c}
N-v \\
j
\end{array}\right) p^{j} \\
& =\left(\begin{array}{c}
N \\
v
\end{array}\right) p^{v}(1-p)^{N-v}
\end{aligned}
$$

and from Eq. (3.23)

$$
\begin{aligned}
P_{v}^{N-v} & \rightarrow \sum_{j=0}^{v}(-1)^{j}\left(\begin{array}{c}
N-v+j \\
j
\end{array}\right)\left[\begin{array}{c}
N \\
N-v+j
\end{array}\right)(1-p)^{N-v+j} \\
& =\left(\begin{array}{c}
N \\
v
\end{array}\right)(1-p)^{N-v} p^{v}
\end{aligned}
$$




\section{FORMULAS FOR $K$-PLUS $L$-SHELL VACANCIES}

\section{A. Subshells not distinguished}

We let $P_{K^{u}, L^{v}}^{K^{2-u}, L^{8-v}}(b)$ be the NEHI probability for producing precisely $u$ vacancies in an initially filled $K$ shell $(u=0,1,2)$ together with precisely $v$ vacancies in an initially filled $L$ shell $(v=0,1, \ldots, 8)$. Then

$$
\begin{aligned}
P_{K^{u}, L^{v}}^{K^{2-u}, L^{8-v}} & \equiv \sum_{\kappa_{1}<\cdots<\kappa_{u} \lambda_{1}<\cdots<\lambda_{v} \mu_{1}<\cdots<\mu_{2-u}} \sum_{v_{1}<\cdots<v_{8-v}} \sum_{\sum_{\kappa_{1}}, \ldots, \kappa_{u}, \lambda_{1}, \ldots, \lambda_{v}} \sum_{\rho_{8-v}}^{\mu_{1}, \ldots, \mu_{2-u}, v_{1}, \ldots, v_{8-v}} \\
& =[u ! v !(2-u) !(8-v) !]^{-1} \sum_{\kappa_{1}, \ldots, \kappa_{u} \lambda_{1}, \ldots, \lambda_{v}, \mu_{1}, \ldots, \mu_{2-u}, v_{1}, \ldots, v_{8-v}} \sum_{\sum} \sum_{\kappa_{1}, \ldots, \kappa_{u}, \lambda_{1}, \ldots, \lambda_{v}}^{\mu_{1}, \ldots, \mu_{2-v}, v_{1}, \ldots, v_{8-v}},
\end{aligned}
$$

where $\kappa_{i}$ and $\mu_{i}$ are spin orbitals of the $K$ shell and $\lambda_{i}$ and $v_{i}$ are those of the $L$ shell. The hyperinclusive expected values are defined by

$$
\begin{aligned}
Q_{K^{u}, L^{v}} & \equiv \sum_{\kappa_{1}<\cdots<\kappa_{u} \lambda_{1}<\cdots<\lambda_{v}} \rho_{\kappa_{1}, \ldots, \kappa_{u}, \lambda_{1}, \ldots, \lambda_{v}} \\
& =[u ! v !]^{-1} \sum_{\kappa_{1}, \ldots, \kappa_{u} \lambda_{1}, \ldots, \lambda_{v}} \rho_{\kappa_{1}, \ldots, \kappa_{u}, \lambda_{1}, \ldots, \lambda_{v}}
\end{aligned}
$$

and

$$
Q^{K^{m} L^{n}}=[m ! n !]^{-1} \sum_{\mu_{1}, \ldots, \mu_{m}} \sum_{v_{1}, \ldots, v_{n}} \rho^{\mu_{1}, \ldots, \mu_{m}, v_{1}, \ldots, v_{n}}
$$

Because the sums over the spin orbitals of the $K$ and $L$ shells are independent of each other, we may apply the results of the previous two sections. Recall that

$$
\begin{aligned}
& P_{K}^{K}=Q_{K}-2 Q_{K^{2}}=Q^{K}-2 Q^{K^{2}}, \\
& P_{K^{2}}^{K^{0}}=Q_{K^{2}}=Q^{K^{0}}-Q^{K}+Q^{K^{2}}\left(Q^{K^{0}}=1\right),
\end{aligned}
$$

and

$$
P_{K^{0}}^{K^{2}}=Q_{K^{0}}-Q_{K}+Q_{K^{2}}=Q^{K^{2}}\left(Q_{K^{0}}=1\right) .
$$

Thus for the " $K$ satellites"

$$
\begin{aligned}
P_{K^{1}, L^{v}}^{K^{1}, L^{8-v}} & =\sum_{j=0}^{8-v}(-1)^{j}\left(\begin{array}{c}
v+j \\
j
\end{array}\right)\left(Q_{K, L^{v+j}}-2 Q_{K^{2}, L^{v+j}}\right) \\
& =\sum_{j=0}^{8-v}(-1)^{j}\left(\begin{array}{c}
8-v+j \\
j
\end{array}\right)\left(Q^{K, L^{8-v+j}}-2 Q^{K^{2}, L^{8-v+j}}\right)
\end{aligned}
$$

in the vacancy and occupancy formalism, respectively. For the " $K$ hypersatellites"

$$
\begin{aligned}
P_{K^{2}, L^{v}}^{K^{0} L^{8-v}} & =\sum_{j=0}^{8-v}(-1)^{j}\left[\begin{array}{c}
v+j \\
j
\end{array}\right) Q_{K^{2}, L^{v+j}} \\
& =\sum_{j=0}^{v}(-1)^{j}\left(\begin{array}{c}
8-v+j \\
j
\end{array}\right]\left(Q^{\left.K^{2}, L^{8-v+j}-Q^{K, L^{8-v+j}}+Q^{L^{8-v+j}}\right) .}\right.
\end{aligned}
$$

And for no $K$-shell vacancies,

$$
\begin{aligned}
P_{K^{0}, L^{v}}^{K^{2}, L^{8-v}} & =\sum_{j=0}^{8-v}(-1)^{j}\left(\begin{array}{c}
v+j \\
j
\end{array}\right)\left(Q_{K^{2}, L^{v+j}}-Q_{K, L^{v+j}}+Q_{L^{v+j}}\right) \\
& =\sum_{j=0}^{v}(-1)^{j}\left(\begin{array}{c}
8-v+j \\
j
\end{array}\right) Q^{K^{2}, L^{8-v+j}} .
\end{aligned}
$$

\section{B. Factorization with respect to projection of intrinsic spin}

In the above formulas the determinants can be as large as $10 \times 10$. Reduction in the number of rows and of columns by a factor of 2 can be obtained if the potentials in the collision problem are independent of the intrinsic spin. Then the transition amplitudes $a_{i j}$ are zero if $i$ and $j$ refer to spin orbitals with different projections of the spin. The determinants $\rho_{i, j}, \ldots$ and $\rho^{i, j, \ldots}$ then factor into products of two determinants, each of which pertains to a specific spin projection. Let $R_{K^{u}, L^{v}}$ (or $R^{K^{m}, L^{n}}$ ) be defined just as is $Q_{K^{u}, L^{v}}$ (or $\left.Q^{K^{m}, L^{n}}\right)$, but with the restriction that all spin orbitals have the same spin projection. We shall assume that each spin-up expected value $R$ equals the corresponding spin-down $R$. Then 


$$
\begin{aligned}
& Q_{K, L^{v}}=2 Q_{1 s \uparrow, L^{v}}=2 \sum_{i} R_{K, L^{i}} R_{L^{v-i}}, \\
& Q_{K^{2}, L^{v}}=\sum_{i} R_{K, L^{i}} R_{K, L^{v-i}},
\end{aligned}
$$

and

$$
Q_{L^{v}}=\sum_{i} R_{L^{i}} R_{L^{v-i}},
$$

where

$$
\max (0, v-4) \leq i \leq \min (4, v) .
$$

The corresponding expressions in the occupancy formalism are exactly the same, with all subscripts raised to superscripts.

The equations of the preceding section for the NEHI probabilities are written in terms of these smaller determinants, $R$, as follows: For precisely one $K$ vacancy,

$$
\begin{aligned}
P_{K, L^{v}}^{K, L^{8-v}} & =2 \sum_{j=0}^{8-v}(-1)^{j}\left(\begin{array}{c}
v+j \\
j
\end{array}\right) \sum_{i} R_{K, L^{i}}\left(R_{L^{v+j-i}}-R_{K, L^{v+j-i}}\right) \\
& =2 \sum_{j=0}^{v}(-1)^{j}\left(\begin{array}{c}
8-v+j \\
j
\end{array}\right) \sum_{k} R^{K, L^{k}}\left(R^{\left.L^{8-v+j-k}-R^{K, L^{8-v+j-k}}\right) .}\right.
\end{aligned}
$$

For precisely two $K$ vacancies,

$$
\begin{aligned}
P_{K^{2}, L^{v}}^{K^{0}, L^{8-v}} & =\sum_{j=0}^{8-v}(-1)^{j}\left(\begin{array}{c}
v+j \\
j
\end{array}\right) \sum_{i} R_{K, L^{i}} R_{K, L^{v+j-i}} \\
& =\sum_{j=0}^{v}(-1)^{j}\left(\begin{array}{c}
8-v+j \\
j
\end{array}\right) \sum_{k}\left(R^{L^{k}}-R^{K, L^{k}}\right)\left(R^{L^{8-v+} \quad{ }^{\prime}}-R^{K, L^{8-v+j-k}}\right)
\end{aligned}
$$

and for precisely no $K$ vacancies,

$$
\begin{aligned}
P_{K^{0}, L^{v}}^{K^{2}, L^{8-v}} & =\sum_{j=0}^{8-v}(-1)^{j}\left(\begin{array}{c}
v+j \\
j
\end{array}\right) \sum_{i}\left(R_{L^{i}}-R_{K, L^{i}}\right)\left(R_{L^{v+j-i}}-R_{K} \quad+j-i\right) \\
& =\sum_{j=0}^{v}(-1)^{j}\left(\begin{array}{c}
8-v+j \\
j
\end{array}\right) \sum_{k} R^{K, L^{k}} R^{K, L^{8-v+j-k}} .
\end{aligned}
$$

Here $i$ is restricted as in Eq. (4.8d) and $k$ satisfies

$$
\max (0,4-v+j) \leq k \leq \min (4,8-v+j) .
$$

The statement of some of these formulas has been given in Ref. 10.

From these number-exclusive probabilities one can obtain the corresponding cross sections by integration over the impact parameter as in Eq. (1.2), i.e.,

$$
\sigma_{K^{u}, L^{v}}^{K^{2-u} L^{8-v}}=2 \pi \int_{0}^{\infty} P_{K^{u}, L^{v}}^{K^{2-u}, L^{8-v}}(b) b d b .
$$

We have used transition amplitudes from our coupledchannel calculations to evaluate these cross sections. Some results have been given. ${ }^{10,12-17} \mathrm{~A}$ detailed presentation of numerical results and comparisons with experimental measurements will be given in a paper which is in preparation.
C. Separation of the $2 s$ and $2 p$ shells

In some $\mathrm{x}$-ray satellite measurements there has been sufficient resolution to subdivide a satellite group with $V$ $L$-shell vacancies into components with $v 2 p$ vacancies and $V-v 2 s$ vacancies. For simplicity we give formulas only for the case of precisely one $K$-shell vacancy and only in the vacancy formalism. The interested reader can obtain the formulas for zero or two $K$ vacancies, or for the occupancy formalism, by analogy with those we have presented. The further distinction between the $L_{\mathrm{II}}$ and $L_{\text {III }}$ subshells requires the use of the $(j, m)$ scheme rather than the $\left(m_{s}, m_{l}\right)$ scheme employed here. We defer a discussion of this case until our computer codes are modified to treat open-shell or relativistic targets. We find, for initially filled $K$ and $L$ shells,

$$
\begin{gathered}
P_{K, 2 s, 2 p^{v}}^{K, 2 s, 2 p^{6-v}}=2 \sum_{j=0}^{6-v}(-1)^{j}\left(\begin{array}{c}
v+j \\
j
\end{array}\right) \sum_{i}\left\{R_{K, 2 s, 2 p^{i}}\left[R_{2 p^{v+j-i}}-R_{K, 2 p^{v+j-i}}-2\left(R_{2 s, 2 p^{v+j-i}}-R_{K, 2 s, 2 p^{v+j-i}}\right)\right]\right. \\
\left.+R_{K, 2 p^{i}}\left(R_{2 s, 2 p^{v+j-i}}-R_{K, 2 s, 2 p^{v+j-i}}\right)\right\}
\end{gathered}
$$




$$
P_{K, 2 s^{0}, 2 p^{v}}^{K, 2 s^{2}, 2 p^{6-v}}=2 \sum_{j=0}^{6-v}(-1)^{j}\left(\begin{array}{c}
v+j \\
j
\end{array}\right) \sum_{i}\left(R_{K, 2 p^{i}}-R_{K, 2 s, 2 p^{i}}\right)\left(R_{2 p^{v+j-i}}-R_{K, 2 p^{v+j-i}}-R_{2 s, 2 p^{v+j-i}}+R_{K, 2 s, 2 p^{v+j-i}}\right)
$$

and

$$
P_{K, 2 s^{2}, 2 p^{v}}^{K, 2 s^{0}, 2 p^{6-v}}=2 \sum_{j=0}^{6-v}(-1)^{j}\left(\begin{array}{c}
v+j \\
j
\end{array}\right) \sum_{i} R_{K, 2 s, 2 p^{i}}\left(R_{2 s, 2 p^{v+j-i}}-R_{K, 2 s, 2 p^{v+j-i}}\right) .
$$

In each of these formulas,

$$
\max (0, v+j-3) \leq i \leq \min (3, v+j) .
$$

\section{APPROACH TO THE BINOMIAL DISTRIBUTION}

Perhaps the main contrast between the IFPM and SPM collision theories is that the IFPM requires the phases of the transition amplitudes connecting all pairs of initially occupied orbitals whereas the SPM requires only singleparticle transition probabilities, which are independent of the phases. Even for an atom as small as neon, to obtain all the needed phases is not a simple assignment. However, our own coupled-channels method, ${ }^{8,13}$ which uses a large target-centered basis, is ideally suited for IFPM multiple-vacancy calculations. We routinely have a good representation in our basis of all the initially occupied target-centered orbitals, and our $U$-matrix procedure for solving the time-dependent coupled equations yields the complex-valued transition amplitudes between all orbitals in the basis in a single computation.

As we have discussed already in preliminary reports of our work, both the experimental and our calculated IFPM $K L^{v}$ multiple-vacancy distributions are in almost all cases very close to binomial distributions given by the SPM. In view of the IFPM formulas presented above this numerical result is at first surprising. The off-diagonal $a_{i j}^{(2)}$ 's are of the same order in the interaction strength as are the diagonal ones which determine the SPM distribution; both are bilinear in the transition amplitudes, $a_{i h}$. There is no formal reason $a$ priori why the off-diagonal parts of the determinants $\rho_{i, j} \ldots$ should be negligibly small. In fact in the case of $K$-shell vacancy production in coincidence with electron transfer to the projectile we found the offdiagonal elements to be quite large. ${ }^{8}$ We have found numerically that the smallness of the off-diagonal $a_{i j}^{(2)}$ 's which enter into multiple-vacancy production is the result of destructive interference in the coherent sums. That is, the individual $a_{i h}^{*} a_{j h}$ may not be small, but their sum over the initially occupied spin orbitals $h$ may be small because of a tendency toward random phases of the amplitudes. ${ }^{10,12,15,16}$

To emphasize this point we note that for projectiles of low charge or high energy, so that the interaction is weak, there is a different way in which the IFPM formulas reduce to the SPM binomial distribution. In Eq. (1.8) or (3.21), if the single-particle vacancy-production amplitudes are small, then $Q_{v}$ will be much larger than $Q_{v+j}$, $j \geq 1$, so $P_{v}^{N-v} \approx Q_{v}$ is a good approximation. With the "average-hole" approximation, $Q_{v} \approx\left(\begin{array}{l}N \\ v\end{array}\right) p^{v}$ (Eq. 3.27), one then has

$$
P_{v}^{N-v} \approx\left(\begin{array}{c}
N \\
v
\end{array}\right] p^{v}, \quad p<1
$$

This is a close approximation to the binomial distribution because for small $p,(1-p)^{n-v} \approx 1$. This approximation of the SPM by the IFPM breaks down, however, for cases with large $p$, where we have found that the IFPM distribution remains nearly binomial, but with a smaller variance. $^{12}$ In these cases it is the random-phase mechanism alone that leads to the nearly binomial distribution.

In summary, we have presented formulas for numberexclusive multiple-vacancy cross sections within the independent-Fermi-particle model. The formulas take complete account of the electron-electron correlations imposed by the Pauli exclusion principle. These NEHI probabilities for producing precisely $u K$-shell and $v L$ shell vacancies are quite different in principle from the hyperinclusive probabilities and expected values for producing at least $v$ vacancies (for which we also provide formulas). The formulas for NEHI probabilities given here have formed the basis for multiple-vacancy calculations ${ }^{10-17}$ that we will report in detail in a subsequent publication. We hope that others will find the concepts and procedures we have developed here useful in deriving analogous results for other cases.

\section{ACKNOWLEDGMENTS}

This research was sponsored at Oak Ridge National Laboratory by the Division of Basic Energy Sciences, U.S. Department of Energy, under Contract No. W-7405-eng26 with Union Carbide Corporation, and at Texas A\&M University by the National Science Foundation under Grant No. PHY-79-09146 and the Center for Energy and Mineral Resources.

\section{APPENDIX: NONITERATIVE INVERSION OF A LINEAR TRANSFORMATION WITH BINOMIAL COEFFICIENTS} by

We shall invert the linear transformation $\vec{x} \rightarrow \vec{y}$ defined

$y_{j}=\sum_{k=0}^{N-j}\left[\begin{array}{c}j+k \\ j\end{array}\right) x_{j+k}=\sum_{i=0}^{N}\left(\begin{array}{l}i \\ j\end{array}\right) x_{i}, j=0,1, \ldots, N$

Observe that for a complex variable $z=w-1$ 


$$
\sum_{j=0}^{N} y_{j} z^{j}=\sum_{i, j=0}^{N}\left(\begin{array}{l}
i \\
j
\end{array}\right) z^{j} x_{i}=\sum_{i=0}^{N} x_{i} w^{i}
$$

But also

$$
\sum_{j=0}^{N} y_{j} z^{j}=\sum_{i, j=0}^{N}(-1)^{i+j}\left(\begin{array}{l}
j \\
i
\end{array}\right) y_{j} w^{i}
$$

By equating the coefficients of the powers of $w$ we have

$$
\begin{aligned}
X_{i} & =\sum_{j=0}^{N}(-1)^{i+j}\left(\begin{array}{l}
j \\
i
\end{array}\right) y_{j} \\
& =\sum_{k=0}^{N-i}(-1)^{k}\left(\begin{array}{c}
i+k \\
i
\end{array}\right) y_{i+k} .
\end{aligned}
$$

This provides a derivation of Eq. (3.21) from Eq. (3.15) or (3.16).
1J. M. Hansteen and O. P. Mosebekk, Phys. Rev. Lett. 29, 136 (1972) (semiclassical Coulomb approximation); J. S. Hansen, Phys. Rev. A $\underline{8}, 822$ (1973); J. H. McGuire and P. Richard, ibid. $\underline{8}, 1374$ (1973) (binary-encounter approximation); J. H. McGuire and L. Weaver, ibid. 16, 41 (1977); R. L. Watson, B. I. Sonobe, J. A. Demarest, and A. Langenberg, ibid. 19, 1529 (1979).

${ }^{2}$ The experimental data and theoretical interpretations are reviewed by P. Richard, in Atomic Inner-Shell Processes, edited by Bernd Crasemann (Academic, New York, 1975), Vol. 1, p. 13; Don H. Madison and E. Merzbacher, in Atomic InnerShell Processes, p. 1; Yohko Awaya, in Electronic and Atomic Collisions, edited by $\mathbf{N}$. Oda and K. Takayanagi (NorthHolland, Amsterdam, 1980), p. 325.

${ }^{3}$ R. L. Becker, J. F. Reading, and A. L. Ford, IEEE Trans. Nucl. Sci. NS-28, 1092 (1981).

${ }^{4} \mathrm{~T}$. Åberg and O. Goscinski, in $X-$ Ray and Atomic Inner-Shell Physics-1982, edited by Bernd Crasemann (American Institute of Physics, New York, 1982), p. 121.

${ }^{5}$ R. L. Becker (unpublished).

6J. F. Reading, Phys. Rev. A $\underline{8}, 3262$ (1973); J. F. Reading and A. L. Ford, ibid. 21, 124 (1980); J. Reinhardt, B. Müller, W. Greiner, and G. Soff, Phys. Rev. Lett. 43, 1307 (1979).

7J. R. MacDonald, C. L. Cocke, and W. W Eidson, Phys. Rev. Lett. 232, 648 (1974); M. Rodbro, E. H. Pedersen, C. L. Cocke, and J. R. MacDonald, Phys. Rev. A 19, 1936 (1979).

${ }^{8}$ R. L. Becker, A. L. Ford, and J. F. Reading, J. Phys. B $\underline{13}$, 4059 (1980); A. L. Ford, J. F. Reading, and R. L. Becker,
Phys. Rev. A 233, 510 (1981).

${ }^{9}$ A. R. Knudsen, D. J. Nagel, P. G. Burkhalter, and K. L. Dunning, Phys. Rev. Lett. 26, 1149 (1971); D. Burch, P. Richard, and R. L. Blake, ibid. 26, 1355 (1971).

${ }^{10}$ R. L. Becker, A. L. Ford, and J. F. Reading, Nucl. Instrum. Methods 214, 49 (1983).

${ }^{11}$ R. L. Becker, A. L. Ford, and J. F Reading, IEEE Trans. Nucl. Sci. NS-30, 1076 (1983).

${ }^{12}$ R. L. Becker, A. L. Ford, and J. F. Reading, in Proceedings of the 3rd International Workshop on Inner-Shell Ionization by Light Ions, Linz, August 1983 [Nucl. Instrum. Methods B $\underline{4}$, No. 2 (1984)].

${ }^{13}$ M. H. Martir, A. L. Ford, J. F. Reading, and R. L. Becker, J. Phys. B 15,2405 (1982), especially p. 2419.

${ }^{14}$ R. L. Becker, J. F. Reading, and A. L. Ford, in Abstracts of the Twelfth International Conference on the Physics of Electronic and Atomic Collisions, Gatlinburg, TN, 1981, edited by S. Datz (North-Holland, Amsterdam, 1981), Vol. 2, p. 852.

${ }^{15}$ R. L. Becker, A. L. Ford, and J. F. Reading, Bull. Am. Phys. Soc. 26, 1309 (1981).

${ }^{16}$ R. L. Becker, A. L. Ford, and J. F. Reading, in International Conference on X-Ray and Atomic Inner-Shell Physics: Program and Abstracts, edited by Bernd Crasemann (Physics Department, University of Oregon, Eugene, 1982), Vol. 1, p. 136.

${ }^{17}$ R. L. Becker, A. L. Ford, and J. F. Reading, Proceedings of the 3rd International Conference on Particle-Induced X-Ray Emission (PIXE) [Nucl. Instrum. Methods (to be published)]. 